\title{
Simulation of Potassium Transport in Carbonate Aquifer
}

\author{
Omar Chaalal ${ }^{1}$, Ahmed Murad ${ }^{2}$, Ahmed M. Soliman ${ }^{3}$, Rafiq Islam ${ }^{5}$, Ismail A. El Haty ${ }^{3}$, \\ D. Hank ${ }^{4}$
}

${ }^{1}$ Chemical and Petroleum Engineering Department, College of Engineering, United Arab Emirates University, Al-Ain, United Arab Emirates

${ }^{2}$ Geology Department, College of Science, United Arab Emirates University, Al-Ain, United Arab Emirates

${ }^{3}$ Chemistry Department, College of Science, United Arab Emirates University, Al-Ain, United Arab Emirates

${ }^{4}$ Nuclear Fuel Technology Department, Hot Labs. Centre, Atomic Energy Authority., Cairo, Egypt

${ }^{5}$ National Polytechnic School of Algiers, Algeria

\section{Email address:}

omar.chaalal@uaeu.ac.ae (O. Chaalal), ahmed.murad@uaeu.ac.ae (A. Murad), a.soliman@uaeu.ac.ae (A. M. Soliman), ismailelhaty@uaeu.ac.ae (I. A. El Haty),d.Hank@hotmail.com (D. Hank)

\section{To cite this article:}

Omar Chaalal, Ahmed Murad, Ahmed M. Soliman, Rafiq Islam, Ismail A. El Haty, D. Hank. Simulation of Potassium Transport in Carbonate Aquifer. American Journal of Environmental Protection. Vol. 4, No. 3, 2015, pp. 127-133. doi: 10.11648/j.ajep.20150403.13

\begin{abstract}
Sophisticated prediction of aquifer performance requires numerical simulation. To date, no comprehensive simulation has been reported on groundwater modeling. Most available simulators are not applicable for fractured aquifer, and do not account for contaminant leaching and degradation, particularly in the vadose zone. Consequently, studying contaminant transport in a fractured or vuggy formation offers a forthidable challenge. This paper addresses the problem of contaminant transport in carbonate aquifer, in the presence of fractures. Most of aquifers in UAE are of limestone or carbonate origins. A series of experiments was conducted using potassium nitrate as the contaminant. Dynamic adsorption and desorption tests were carried out using both homogeneous and fractured formation models. Initial modeling and experiments were carried out for a range of initial concentration values. The concentration at the outlet was measured with the Flame Ionization technique. A numerical model was developed using the surface excess theory, combined with a non-Fickian dispersion coefficient. Numerical results agreed favorably with experimental results. It was found that the non-Fickian model was necessary for modeling fracture flow results and with this version, there was no need to use the dual porosity/dual permeability formulation. Strong dependence of adsorption on initial concentration was observed and was justified with the numerical model.
\end{abstract}

Keywords: Carbonate Aquifer, Modling, A non-Fickian Dispersion Coefficient, Potassium Nitrate, Dual Porosity and Numerical Simulation

\section{Introduction}

In the past three decades, fractured rock domains have received increasing attention by the researchers from a number of disciplines, including hydrogeology, petroleum engineering, and environmental engineering. The importance of fractures is particularly enhanced when one deals with carbonate formations, both in water flow and petroleum production, because most of these formations are known to be fractured. The subject has been investigated by the petroleum engineers in connection with multiphase flow, because many important petroleum reservoirs are in fractured rock formations. Of special interest are reservoirs composed of fractured porous rocks in which the matrix blocks, surrounded by the network of fractures are porous. The permeability of such blocks are often rather low, but the porosity and, hence, the storage capacity for fluids are very high.

Environmental engineers have been investigating the problem in connection with geological isolation of radioactive waste and remediation of ground water. Hydrologists deal with fractured formations because numerous deep aquifers are fractured. Chemical engineers mainly deal with chemical transport in limestone, but not necessarily in its fractured form (Arsic et al., 1991; Couturier et al., 1993).

In Canada, Switzerland, and Sweden large national research programs are underway to study radio-nuclide transport in crystalline rocks. Finland also intends to site their repository in similar rocks and has a research program directed toward this end. Other countries, including Japan, France, Spain, the United Kingdom, and the USA have or 
have had research programs oriented towards crystalline rocks. In addition, an international field of research program is in progress in the Stripa mine, Sweden. The research is aimed at understanding the processes that determine the flow paths and the flow rates of water in the fractured rock masses.

Solutes that are dissolved in water will be carried by the moving water, but various other mechanisms are involved in the process of solute transportation. Small molecules or ions diffuse in a concentration gradient and can move from one 'stream tube' to another.

Different water volumes move with different velocities and may mix at more or less regular intervals. The dissolved species may also experience kinetic effects caused by physical processes. One of such processes that may have a large impact for solute transport in fractured rock is the diffusion in and out of zones in which the water is moving so slowly that it can, for all practical purposes, be assumed to be stagnant. Such stagnant zones can be expected in fractures with uneven surfaces and with fracture-filling materials. In rocks with a connected matrix can be very much larger than the mobile water volume in the fracture. Because the stagnant zones are reached by diffusion, the volume of stagnant water accessed depends on the residence time of the flowing water.

Different approaches to solute transport in fractured media have been attempted. These include the analysis of a single fracture (e.g. Grisak and Pickens, 1981; Tang et al., 1981; Sudicky and Frind, 1984). While some researchers considered the porous medium as comprised of equallyspaced fractures (Sudicky and Frind, 1984), others have used deterministic study of flow in an irregular network of fractures contained in an impermeable rock. Yet others (Schwartz and Smith, 1988) considered the same problem with a stochastic model. Recently, Choi et al. (1997) used a dual-permeability, dual-porosity approach to model fluid flow through a fractured formation. They built their model using non-Darcian flow through the fracture while allowing Darcian flow through the matrix. Even though this work was devoted to advancing the hydrodynamic aspect of fluid flow, no species transport was considered. Adsorption and desorption are important phenomena in chemical transport. A Langmuir isotherm has been used most commonly to model adsorption at the solid/liquid interface. The widespread use of the Langmuir model arises from its ability to represent the general shape of many adsorption isotherms. However, more recently, the surface excess theory has been proven to be more theoretically sound and better apt in modeling adsorption (Islam and Chakma, 1991).

Furthermore, Sarwar and Islam (1997) demonstrated that the surface excess theory is also capable of representing chemical transport through fractured formations. However, non-Fickian dispersion had to be introduced, along with nonequilibrium adsorption and desorption rates. They considered the transport several chemicals in silica sand formation and observed very good agreement between experimental and numerical results, even for the regime with non-uniform adsorption and desorption profiles. Recently Zargham et al
(2011) have described the adsorption behavior in a system of solid phase carbonate and of an aqueous phase of surfactant. They found that the rate of adsorption dependents on availability of surfactant in the system and the adsorption of surfactant increased with increasing surfactant concentration. This research comprises of laboratory flow experiments and numerical modeling of $\mathrm{KNO} 3$ transport in carbonate formations. Such flow is of relevance to agricultural applications for which run-offs from fertilizers constitute major contamination problems for groundwater. Also, in petroleum engineering applications, mathematical models of chemical transport are of importance, especially for the case of fractured carbonate formations.

\section{Experimental Setup and Procedure}

A glass column was used for all experimental tests. Because only hydrostatic pressure was used to maintain flow through the porous medium, little pressure was anticipated in the column and a glass column was sufficient. Natural carbonate rocks were collected from the outcrops of Al-Ain (near Jabel Hafeet). These samples were crushed in order to facilitate packing of this material in the column. The porosity of the crushed rock was approximately $15 \%$. This was considered to be the result of adequate packing. The packed column was connected to an inlet (for injection of the contaminated water) and was released to the outside at atmospheric pressure. A two-folded aluminum foil was placed parallel to the axis of the cylinder to model a single fracture. The foil contains approximately 50 holes (performed with a $0.05 \mathrm{~mm}$ needle) per $\mathrm{cm}^{2}$, in order to assure cross flow as well as fracture flow. This was the procedure previously implemented by Sarwar and Islam (1997).

The Nitrate used was a solution of $\mathrm{KNO}_{3}$. This form of the potassium is considered to be difficult to remove from a water stream. Two different concentrations of nitrite, 50 and $150 \mathrm{ppm}$, were used in this study. Standard solutions were prepared using laboratory-grade $\mathrm{KNO}_{3}$. Deionized water was used for all the experiments, as a solvent (adsorption tests) as well as a washing agent (desorption tests).

\subsection{Procedure}

In order to observe both adsorption and desorption, contaminated water was injected in each sample and was followed by fresh water after a high number of pore volumes of fluid injection. During this process, samples were collected at the outlet and were analyzed for potassium content in the effluent. The concentration at the outlet was measured with a Digital Flame Analyzer (Model 2655000, as supplied by Cole Palmer Instrument Co., Chicago, USA). This instrument was made available by the Water and Electricity Laboratory of Al-Ain in UAE.

\subsection{Theory}

Adsorption of a chemical is considered to take place from 
the monomer phase. Surface excess is the measure of adsorption from liquid mixtures that describes the difference between the actual amount of component $i$ adsorbed and the amount of component $i$ that would be present in the adsorbed phase if the composition and the density of that phase were same as those of the phase in equilibrium. For a binary system, the surface excess of component $i$ is given by:

$$
n_{i}^{e}=n^{o}\left(x_{i}^{o}-x_{i}\right)
$$

where $i=1$ denotes chemical $(i=1)$ and $i=2$ denotes the bulk phase carrying the chemical. If it is assumed that the liquid phase is directly in contact with the solid, the above equation assumes the following form

$$
n_{i}^{e}=n^{\prime}\left(x_{i}^{\prime}-x_{i}\right)
$$

Using the monomer adsorption model, the surface excess can be expressed as

$$
\frac{1}{n^{\prime}}=\frac{x_{1}^{\prime}}{m_{1}}+\frac{x_{2}^{\prime}}{m_{2}}
$$

where $m_{1}$ and $m_{2}$ represent the amounts of monolayer coverage for the chemical and the bulk phase, respectively. At this point the concept of selectivity can be introduced. Selectivity, $S$, is defined as follows:

$$
S=\frac{x_{1}^{\prime} / x_{2}^{\prime}}{x_{1} / x_{2}}
$$

Even though the monolayer model is based on the assumption of monolayer adsorption, it has been taken for granted that the monolayer model is also suitable for multilayered adsorption (Basu et al. 2006; Song and Islam, 1994). With selectivity, the expression of surface excess can be written as

$$
n_{a}^{e}=\frac{m_{1} x_{1} x_{2}(S-1)}{S x_{1}+\left(m_{1} / m_{2}\right) x_{2}}
$$

The amount of chemical adsorbed is given by

$$
n_{1}^{\prime}=\frac{m_{1} x_{1} S}{S x_{1}+\left(m_{1} / m_{2}\right) x_{2}}
$$

Finally, the kinetic term is calculated as:

$$
\frac{\partial n_{1}^{e}}{\partial i}=k_{i}\left(n_{1}^{e}-n_{1}^{e a}\right)
$$

where $\mathrm{k} i=\mathrm{k}_{1}$ for desorption. This term introduces the possibility of desorption of a chemical in a dynamic displacement system.

\section{Governing Equation}

The one-dimensional mass transfer equation that includes the surface excess theory to describe adsorption in the solid/liquid interface is given as (Song and Islam, 1994):

$$
\frac{\lambda q \rho_{1}}{A \phi} \frac{\partial^{2} C}{\partial z^{2}}-\frac{q \rho_{1}}{A \phi} \frac{\partial C}{\partial z}=\frac{1-\phi}{\phi} \rho_{r} n_{o} \frac{\partial C}{\partial i}+\frac{1-\phi}{\phi} \rho_{r}\left(\frac{\partial n_{1}^{e a}}{\partial i}\right)
$$

\section{Numerical Solution Method and Accuracy}

In order to discretize Eq. (8) the numerical scheme of Barakat and Clark (1966) was used. This scheme has been proven to be powerful in solving diffusive convection equation in the past. Numerical simulation was carried out in a linear grid system of 100 grid blocks. This number was selected after a series of trial runs for optimum accuracy. Time was discretized with a $\Delta t$ of 50 seconds. This value was found to be appropriate considering that the spatial grid blocks were rather small. The following boundary and initial conditions were used:

$$
C(z, 0)=0 \text {, for } 0 \leq \mathrm{z} \leq \mathrm{L}
$$

$C(0, t)=C_{o}+\lambda(\partial C / \partial z)$, for the adsorption test. For desorption, $\mathrm{C}_{\mathrm{o}}$ is set to zero.

$$
\left.\frac{\partial C}{\partial z}\right|_{z=L}=0, \text { for } \mathrm{t}>0
$$

Table 1. Input Data

\begin{tabular}{llll}
\hline $\mathbf{C}_{\mathbf{o}}$ & $\mathbf{5 0 , 1 0 0} \mathbf{~ p p m}$ & $\boldsymbol{\lambda}$ & Variable, see Table 2 \\
\hline$K$ & $11.3 \mathrm{mD}$ & $\mathrm{m}_{1} / \mathrm{m}_{2}$ & 15 \\
$\mathrm{n}_{\mathrm{o}}$ & $200 \mathrm{mg} / \mathrm{g}$ & $\mathrm{S}$ & 25 \\
$Q$ & $10 \mathrm{ml} / \mathrm{min}$ & $m_{1}$ & $0.05 \mathrm{n}_{\mathrm{o}}$ \\
$\Phi$ & 0.15 & $k_{1}$ & $2 / \mathrm{hr}$ \\
$\rho_{\mathrm{i}}$ & $1 \mathrm{~cm}^{3} / \mathrm{ml}$ & $k_{2}$ & Variable, see Table 2 \\
$\rho_{\mathrm{r}}$ & $2.8 \mathrm{~g} / \mathrm{ml}$ & $\mathrm{PV}$ & $22 \mathrm{~cm}^{3}$ \\
\hline
\end{tabular}

Numerical simulation runs were conducted in order to obtain best match between experimental and numerical results. The best match data are reported in Table 1. Note that the dispersion parameter was found to be a function of concentration. This aspect will be discussed later.

\section{Experimental Results and Discussion}

Eight experimental runs were conducted with $\mathrm{KNO}_{3}$ as the chemical with two different concentrations (50 and 150 $\mathrm{ppm})$. Description of each run is given in Table 2. For each concentration, four runs were conducted in absence or presence of fracture and adsorption and desorption. Following is a discussion of various experimental runs.

Figure 1 shows the results of Runs 1 and 2 (adsorption and desorption with $50 \mathrm{ppm}$ solution). These two runs were conducted on the same porous material. Adsorption test was simply followed by the desorption test that involved the injection of de-ionized water. As can be seen from Fig. 1, the outlet concentration rose to the same level as that of the inlet only after the injection of 10 pore volumes (PV) of water. This value is relatively higher as compared to the case using silica sand, for which saturation (to a dimensionless concentration of unity) was reached within two pore volumes of chemical injection (Sarwar and Islam, 1997). Indeed, carbonate formations exhibit much higher adsorptive capacity than do silica sands. Crushed carbonate has much greater surface area 
than silica sand. This is reflected in the fact that the permeability of crushed carbonate is several hundred times smaller than that of silica sand. The outlet concentration is measured in terms of potassium content. It was not possible in this study to determine the fate of $\mathrm{NO}_{2}^{-}$in this study. It might be interesting to find the partition of $\mathrm{NO}_{2}^{-}$in the solid and the liquid phases. The desorption trail in Fig. 1 shows much faster decline in outlet concentration than that of the adsorption curve. This was in contrast to silica sand for which slower desorption was observed (Sarwar and Islam, 1994). However, only limited amount of chemical was injected in their case and most likely nothing more than a monomer layer was allowed to be formed.

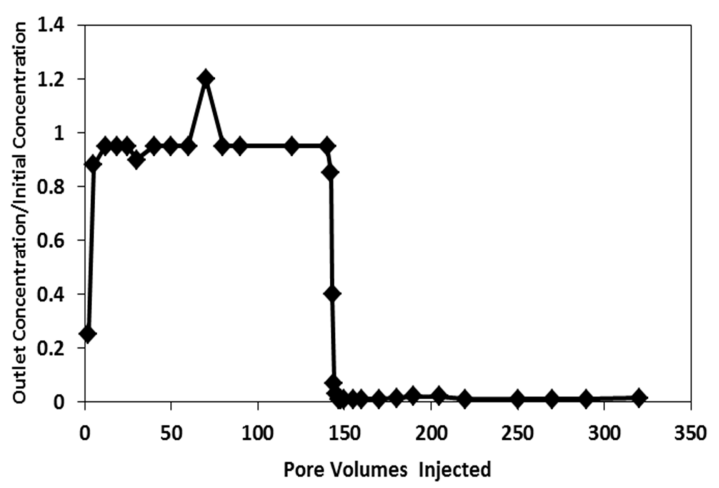

Fig. 1. Chemical adsorption and desorption in absence of fracture (initial concentration $=50 \mathrm{ppm}$ ).

Figure 2 shows the adsorption and desorption profiles of Runs 3 and 4, respectively. These runs were conducted with $50 \mathrm{ppm}$ nitrite solution during the adsorption phase. However, a single fracture was added to the system. This resulted in a smaller pressure drop across the porous medium in order to maintain the same flow rate as the homogeneous case (Fig. 1). However, the chemical transport was not particularly affected by the presence of the fracture. The saturation (the point of reaching concentration ratio of unity) took place at approximately the same time as that of the homogeneous case. It is conceivable that fractures will play a greater role at higher flow rates. This aspect of the study is currently underway.

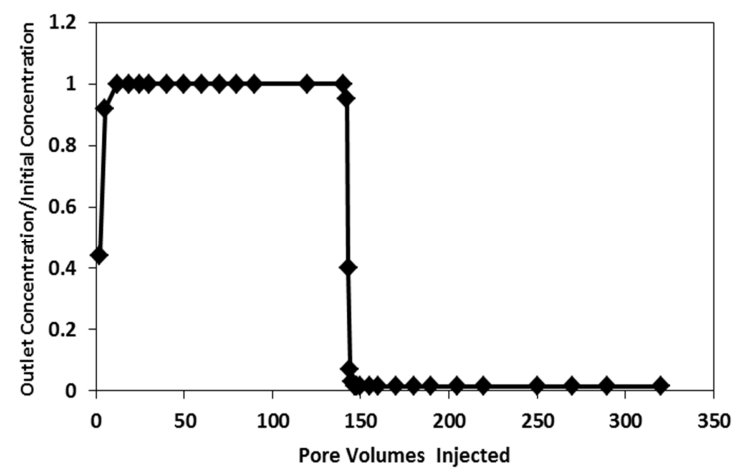

Fig. 2. Chemical adsorption and desorption in presence of fracture (initial concentration $=50 \mathrm{ppm}$ ).

Figure 3 shows adsorption and desorption results of Runs
3 and 4 that were conducted with $150 \mathrm{ppm}$ solution. The adsorption appears to reach steady state at a much slower pace than the one reached by lower concentration cases. Adsorption indeed depends on the concentration gradient that is likely to be higher in this case than in the case of lower absolute concentration. This effect, however, cannot be observed unless a dynamic test is performed as done in this study. The effect of concentration on desorption is less pronounced.

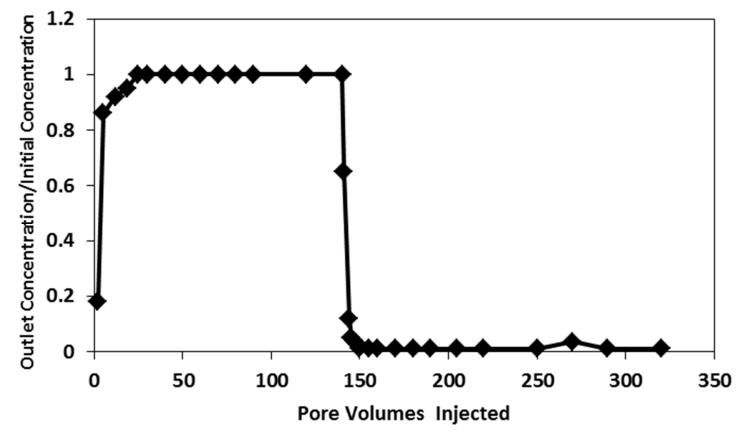

Fig. 3. Chemical adsorption and desorption in absence of fracture (initial concentration $=150 \mathrm{ppm}$ ).

Figure 4 shows the adsorption and desorption profiles for the $150 \mathrm{ppm}$ in presence of a fracture. For this case, the effect of fracture is clearly shown. The whole adsorption curve is moved to the left, in effect showing that chemical transport remain faster than the homogeneous case. Also, fractures play a greater role in the presence of higher concentration of the contaminant. This contribution of fractures can be modeled by non-Fickian dispersion (Sarwar and Islam, 1994). It is also conceivable that the adsorption and desorption rates are also affected. This will be discussed in the following section on numerical modeling.

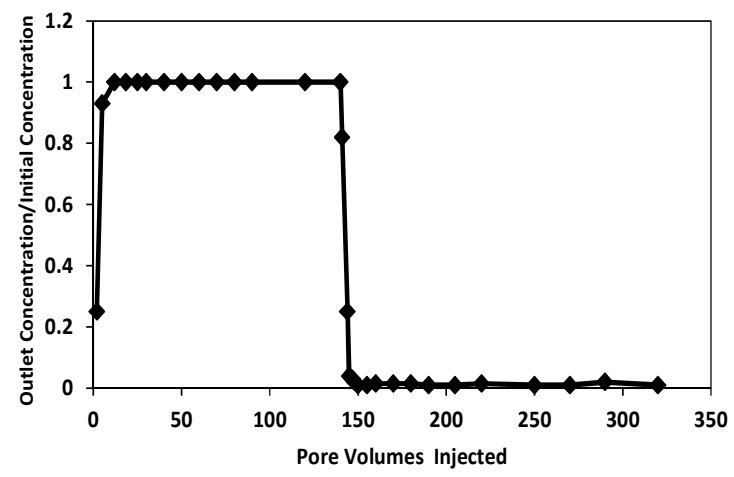

Fig. 4. Chemical adsorption and desorption in presence of fracture (initial concentration $=150 \mathrm{ppm}$ ).

\section{Discussion on Mathematical Modeling}

Numerical simulation runs were conducted individually for each section of adsorption and desorption separately. This helped determine the adsorption and desorption rates independently. Following is a discussion on the mathematical modeling results. 
Figure 5 compares numerical and experimental results of Run 1 (adsorption with $50 \mathrm{ppm}$ solution, in absence of fracture). The agreement between experimental and numerical results is excellent. Resulting best-fit parameters are listed in Table 1. Numerical modeling of such a process is further consolidated if the breakthrough values could be monitored. Unfortunately, breakthrough values were not monitored in the experimental study.

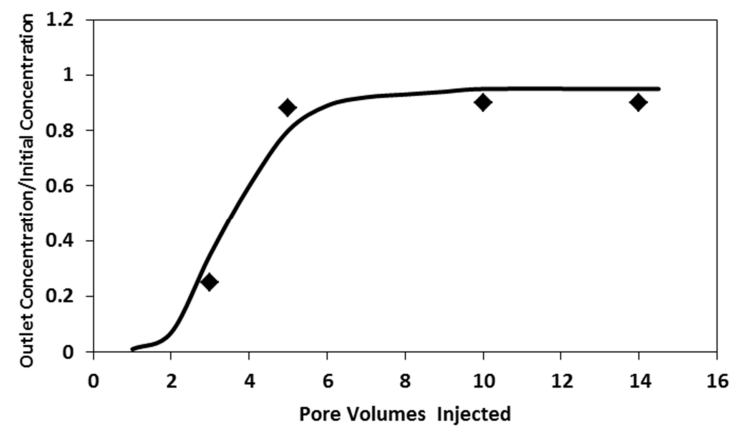

Fig. 5. Comparison of experimental and numerical results of an initial concentration of $50 \mathrm{ppm}$ (no fracture).

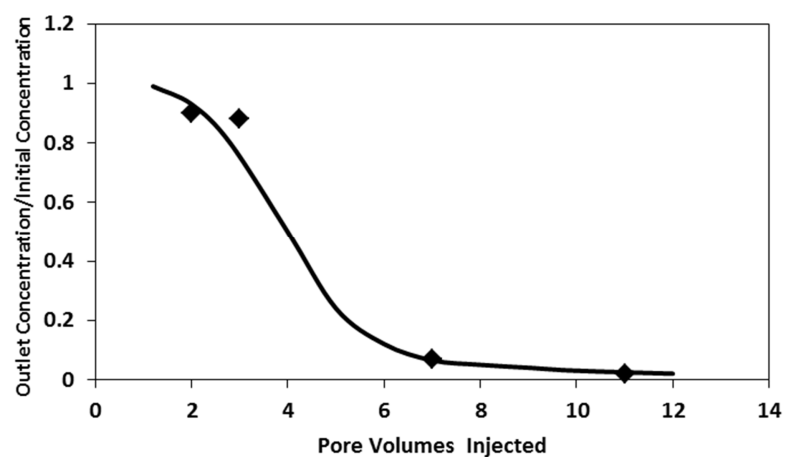

Fig. 6. Comparison of experimental and numerical results of an initial concentration of $50 \mathrm{ppm}$ (no fracture).

The experimental and numerical results of Run 2 (desorption with $50 \mathrm{ppm}$ solution in absence of a fracture) are shown in Fig. 6. A much lower desorption rate compared to adsorption rate had to be used in order to match the experimental desorption data. It commonly found that the adsorption rate is higher than the desorption rate. This, in fact, forms the basis for irreversible thermodynamics and the surface excess theory. In order to obtain a better match between experimental and numerical results, the dispersion parameter used in the desorption phase was somewhat smaller than that of the adsorption phase. It is expected that the dispersion of fresh water into contaminated water (desorption test) be different than the dispersion of contaminated water into fresh water (adsorption test). This process is further complicated by the presence of tightly packed crushed carbonate rocks. No other variable was adjusted in order obtain the match between experimental and numerical results. Also, these numbers are significantly different from those of silica sand (Sarwar and Islam, 1994). Note, however, that the concentration of nitrate used by Sarwar and Islam was much lower than the one used in this study.

Figure 7 compares experimental and numerical results of Run 3. For this run, an adsorption test was performed using a concentration of $150 \mathrm{ppm}$, in absence of fractures.

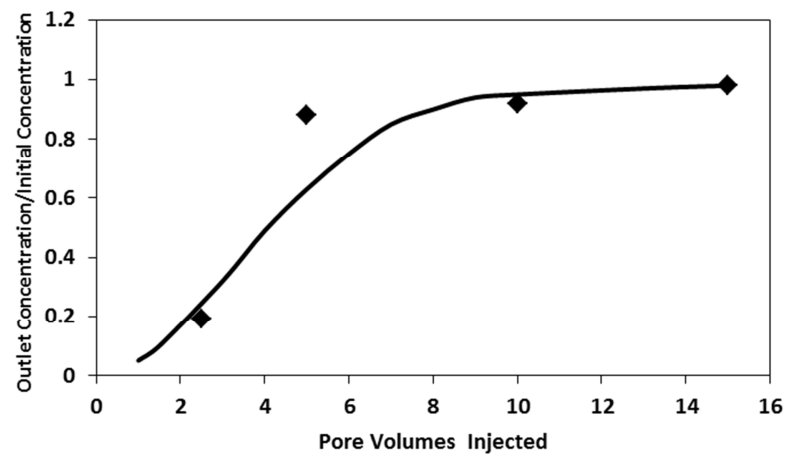

Fig. 7. Comparison of experimental and numerical results of an initial concentration of $150 \mathrm{ppm}$ (no fracture).

For this numerical run no parameter was adjusted. Note that the agreement between experimental and numerical results is remarkably good, especially considering that no parameter was adjusted. In order to obtain a good match between experimental and numerical results of the desorption test, the dispersion rate was first modified. This alone did not improve the agreement that showed elongated desorption trail. In order to remedy this discrepancy, the rate of desorption was modified (see Table 2). It is not unusual to find that the desorption rate, be a function of concentration (Sarwar and Islam, 1994). It is particularly important to realize that at higher concentrations, the assumption of monolayer may not applicable, in which case desorption will depend on the concentration, rather than on the surface properties of the solid. Figure 8 shows that the agreement between experimental and numerical results is greatly improved by modifying the desorption rate.

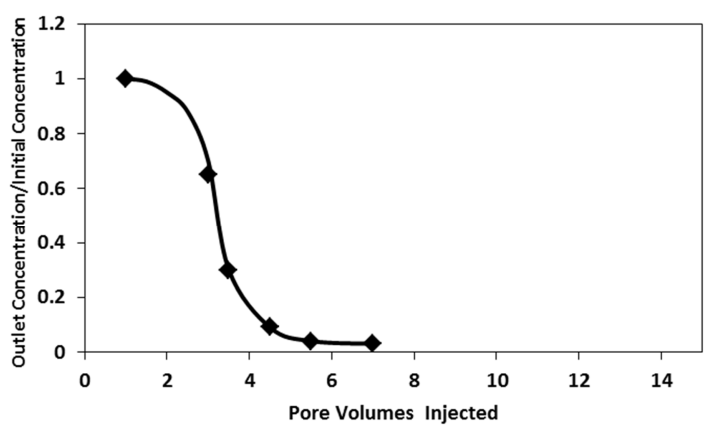

Fig. 8. Comparison of experimental and numerical results of an initial concentration of $150 \mathrm{ppm}$ (no fracture).

Figure 9 compares experimental and numerical results of Run 5 for which $50 \mathrm{ppm}$ solution was used to conduct the adsorption test in presence of a single fracture. Excellent agreement between experimental and numerical results is obtained only after modifying the dispersion rate to one third of that of the previous adsorption tests. The presence of 
fracture invokes an increase in permeability, and hence creates a channel for chemical transport. This can result in decreased adsorption (due to bypassing of chemicals) or a decrease in dispersion (as convection is affected by an increase in permeability). Note that the adsorption or desorption rates were not changed due to the presence of the fracture. An excellent agreement between experimental and numerical results can be seen in Fig. 10. The agreement is particularly good during the quick change in outlet concentration for during which the dynamic phenomena are most active. Considering that only the dispersion parameter was adjusted for this desorption case, the agreement between experimental and numerical results is impressive.

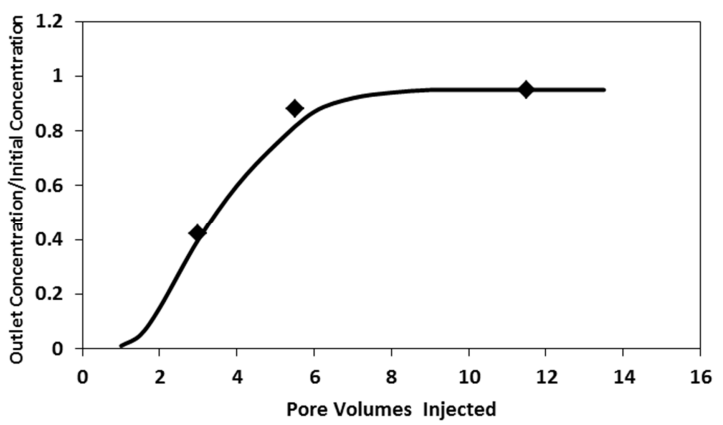

Fig. 9. Comparison of experimental and numerical results of an initial concentration of $50 \mathrm{ppm}$ (with fracture).

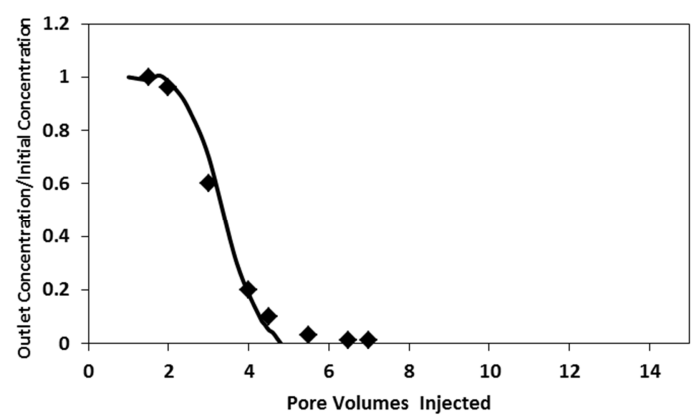

Fig. 10. Comparison of experimental and numerical results of an initial concentration of $50 \mathrm{ppm}$ (with fracture).

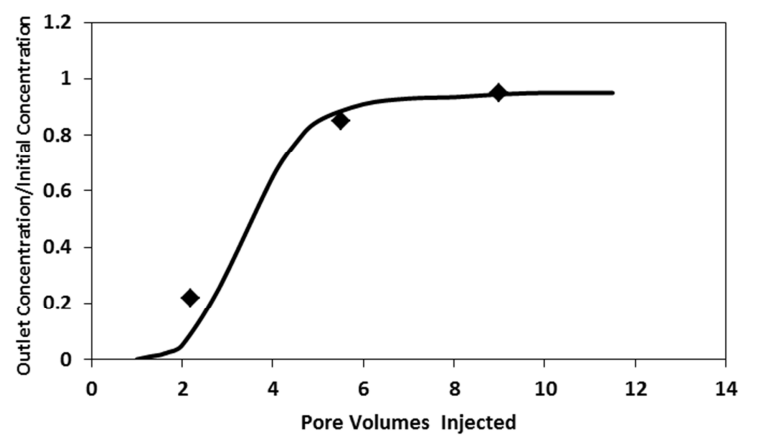

Fig. 11. Comparison of experimental and numerical results of an initial concentration of $150 \mathrm{ppm}$ (with fracture).

Figure 11 shows the comparison between experimental and numerical for Run 7 that was conducted with a $150 \mathrm{ppm}$ solution using a fractured formation. Figure 12 shows the same for the desorption case. Note that the agreement between experimental and numerical results is obtained without making any adjustment to the adjustable parameters (see Table 2).

Table 2. Experimental Runs

\begin{tabular}{llll}
\hline Run no. & $\boldsymbol{\Lambda}$ & $\mathbf{K}_{\mathbf{2}}$ & Description of runs \\
\hline 1 & 3 & 0.03 & $50 \mathrm{ppm}$, adsorption test, no fracture \\
2 & 1.1 & 0.03 & $50 \mathrm{ppm}$, desorption test, no fracture \\
3 & 3 & 0.03 & $150 \mathrm{ppm}$, adsorption test, no fracture \\
4 & 0.25 & 0.01 & $150 \mathrm{ppm}$, desorption test, no fracture \\
5 & 1 & 0.03 & $50 \mathrm{ppm}$, adsorption test, with fracture \\
6 & 0.1 & 0.03 & $50 \mathrm{ppm}$, desorption test, with fracture \\
7 & 1 & 0.03 & $150 \mathrm{ppm}$, adsorption test, with fracture \\
8 & 0.1 & 0.01 & $150 \mathrm{ppm}$, desorption test, no fracture \\
\hline
\end{tabular}

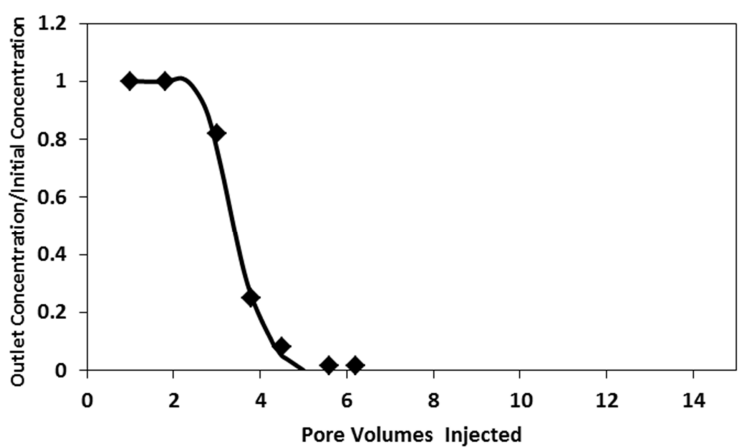

Fig. 12. Comparison of experimental and numerical results of an initial concentration of $150 \mathrm{ppm}$ (with fracture).

\section{Conclusions}

A series of experimental and numerical runs was conducted in order to describe nitrite flow in a carbonate formation with or without the presence of a linear fracture. Experimental results show that the kinematics of adsorption and desorption depend on the concentration of the solute, leading to the use of a non-Fickian dispersion model. This model is coupled with the surface excess theory and showed excellent agreement with experimental results with minimum number of adjustable parameters.

\section{Acknowledgments}

Partial funding of this research was provided by the United Arab Emirates University [College of Science (21S065COS/IRG-09/13)]

\section{Nomenclature}

$A=$ cross sectional area of the core, $\mathrm{cm}^{2}$ $C=$ concentration of the chemical, $\% \mathrm{wt}$

$\mathrm{C}_{\mathrm{o}}=$ concentration of the chemical , \%wt

$k_{\mathrm{i}}=$ kinetic constant of adsorption $(i=1)$ or desorption $(i=2)$, $1 / \mathrm{hr}$

$\mathrm{k}=$ absolute permeability of the porous medium, $\mathrm{mD}$ 
$L=$ length of the porous bed, $\mathrm{cm}$

$\mathrm{m}_{\mathrm{i}}=$ monolayer coverage of component $\mathrm{i}$ per unit of adsorbent, $\mathrm{mg} / \mathrm{g}$

$\mathrm{n}^{\circ}=$ amount of liquid per unit mass of adsorbent, $\mathrm{mg} / \mathrm{g}$

$\mathrm{n}^{\prime}=$ amount adsorbed per unit mass of adsorbent, $\mathrm{mg} / \mathrm{g}$

$n_{i}^{\prime}=$ amount of component $\mathrm{i}$ adsorbed per unit mass of adsorbent, $\mathrm{mg} / \mathrm{g}$

$n^{e}{ }_{\mathrm{i}}=$ equilibrium surface excess of component $\mathrm{i}$ per unit mass of adsorbent, $\mathrm{mg} / \mathrm{g}$

$n_{i}^{e a}=$ actual surface excess of component i per unit mass of adsorbent, $\mathrm{mg} / \mathrm{g}$

$\mathrm{PV}=$ pore volume, $A \phi L, \mathrm{~cm}^{3}$

$q=$ flow rate, $\mathrm{ml} / \mathrm{hr}$

$S=$ selectivity, dimensionless

$\mathrm{t}=$ time, $\mathrm{s}$

$\mathrm{u}=$ local speed, $\mathrm{cm} / \mathrm{s}$

$x_{i}=$ bulk phase mass fraction of component $\mathrm{i}$

$x_{i}^{\prime}=$ adsorbed phase mass fraction of component $\mathrm{i}$

$z=$ distance along porous bed, $\mathrm{cm}$

$a=$ entrainment rate constant, $1 / \mathrm{cm}$

$\lambda=$ dispersion parameter, $\mathrm{cm}$

$\phi=$ porosity of the rock

$\rho_{\mathrm{i}}=$ liquid density, $\mathrm{mg} / \mathrm{ml}$

$\rho_{\mathrm{r}}=$ rock density, $\mathrm{g} / \mathrm{m} 1$

\section{References}

[1] Arsic, B., Oka, S., and Radovanovic, M., 1991, "Characterization of Limestones for $\mathrm{SO} 2$ Absorption in Fluidized Bed Combustion", FBC Technology and the Environmental Challenge, Hilger, London, p. 171.

[2] Barakat, H.Z. and Clark, J.A., 1966, "On the Solution of the Diffusion Equations by Numerical Methods", ASME Trans. Heat Transfer, vol. 88,83 .

[3] Basu, A. , Mustafiz, S., Islam, M. R., Bjordalen, N., Rahman M. S. and Chaalal, O. 2006, "A Comprehensive Approach for Modeling Sorption of Lead and Cobalt Ions through Fish Scales as an Adsorbent" Chem. Eng. Comm., 193:580-605.
[4] Choi, E.S., Cheema, T.J., and Islam, M.R., 1997, "A New dual porosity/dual permeability model with non-Darcian flow through fractures", J. Petroleum Science and Engineering, vol. $17,331-344$.

[5] Couturier, M.F., Karidio, I., and Steward, F.R., 1993, "Study on the Rate of Breakage of Various Canadian Limestones in a Circulating Transport Reactor", Circulating Fluidized Bed Technology IV, A.A. Avidan, ed., Amer. Inst. Chem. Eng., New York, p. 672.

[6] Islam, M.R., and Chakma, A., 1991, "Mathematical Modelling of Enhanced Oil Recovery by Alkali Solutions in the Presence of Cosurfactant and Polymer", J. Pet. Sci. Eng., vol. 5, 105126.

[7] Sarwar, M., and Islam, M.R., 1997, "A Non-Ficician Surface Excess Model for Chemical Transport Through Fractured Porous Media", Chem. Eng. Comm., vol. 160, 1-34.

[8] Schwartz, F.W., and Smith, L., 1988, "A Continuum Approach for Modeling Mass Transport in Fractured Media", Water Resources Res., vol. 24 (8), 1360-1372.

[9] Song, F.Y. and Islam, M.R., 1994, "Effect of Salinity and Rock Type on Sorption Behavior of Surfactants as Applied in Cleaning of Petroleum Contaminants", J. Pet. Sci. Eng., vol. 10 (4), 321-336.

[10] Sudicky, E.A., and Frind, E.O., 1982, "Contaminant Transport in Fractured Porous Media: Analytical Solution for a Single Fracture", Water Resources Res., vol. 21 , 1677-1683.

[11] Tang, D.H., Frind, E.O., and Sudicky, E.A., 1981, "Contaminant Transport in Fractured Porous Media: Analytical Solution for a System of Parallel Fractures", Water Resources Res., vol. 17 (3), 555-564.

[12] Zargham Salari, Mohammad Ali Ahmadi, Riaz Kharrat and Abbas Abbaszadeh Shahri 2011 "Experimental Studies of Cationic Surfactant Adsorption onto Carbonate Rocks" Australian Journal of Basic and Applied Sciences, 5(12): 808813. 